\title{
Improving room layouts for venepuncture, cannulation and ABG equipment on surgical wards
}

Ryan Pedley, Anna Whitehouse, Sarah Hammond

St George's Hospital NHS Trust

\begin{abstract}
The Productive Ward series has effectively helped to standardise the storage of equipment in hospital ward treatment rooms; however, in our organisation equipment for venepunture and cannulation had been excluded. This resulted in clinicians having to navigate several unfamiliar environments while on-call and hence waste valuable time searching for basic equipment.
\end{abstract}

We aimed to make it easier to locate and identify the basic equipment used for cannulation, venepuncture, and arterial blood gas sampling and more efficient to collect. We examined the initial layout of equipment on four surgical wards in a large teaching hospital. The time taken for junior doctors, nurses, health care assistants, and physician assistants to gather equipment on these wards was recorded along with a process map of steps involved. Our intervention was to relocate the equipment into adjacent storage and make it easily identifiable by the use of a 'red dot'. Following these changes we repeated the measurements.

There was an overall reduction in the mean time taken to gather the equipment required to insert a venous cannula on an unfamiliar ward from 2 min 41 s pre-intervention (range $52 \mathrm{~s}$ to 6 mins $58 \mathrm{~s}, \mathrm{n}=23$ ) to $26 \mathrm{~s}$ post-intervention (range $8 \mathrm{~s}$ to $1 \mathrm{~min} 20 \mathrm{~s}, \mathrm{n}=51$ ). Additionally, the number of steps involved in the process was reduced from 16 to five. All of the 32 junior doctors surveyed felt that faster identification improved patient safety.

A significant reduction in the time wasted by clinicians searching for venepuncture equipment on surgical wards has been achieved by simplifying the storage, layout, and identification of this kit. The accumulated benefit includes increased productivity, familiarity, and safety, which is paramount when attending unwell patients on unfamiliar wards.

\section{Problem}

Most hospital wards contain a similar set of standardised equipment. Despite this, the layout of each ward can be highly variable, making locating equipment time-consuming. This is particularly relevant for the on-call clinician, who covers large, unfamiliar areas of the hospital and can be responsible for a higher number of unwell patients.

In the non-acute setting, this wastes time that could be spent with patients; in the acute setting, these time delays could significantly compromise patient care.

We set out to standardise commonly used equipment on hospital wards. We focused on items for phlebotomy, cannulation, and arterial blood gas (ABG) sampling.

\section{Background}

In the most acute of situations, during in-hospital cardiac arrests, time delays in delivery of emergency treatment have been shown to be directly detrimental to patient care in terms of reduced survival to discharge (1). These factors can be separated into patient and hospital related factors, which include time taken to access emergency equipment. Accordingly, steps have been taken and recommendations made to ensure a standardisation of emergency equipment and drugs in cardiac arrest trolleys (2). These principles can be extrapolated to other areas. Delays locating equipment could waste clinician's valuable time and cause treatment delays.

A wide variety of equipment is required to perform simple procedures such as phlebotomy, cannulation, and ABG sampling. Other sites have seen significant discrepancies between the layouts of different surgical wards within the same hospital ( $E$ Andrews, $J$ Bickley, H Blakey, B Gibbons, J Wynter Bee. Standardising the treatment rooms at Croydon University Hospital. Croydon University Hospital; unpublished). The individual items we chose to look at included: tourniquets, alcohol swabs, dressing, saline flush, syringes $(10 \mathrm{ml})$, needles (hypodermic and butterfly), blood bottles (for biochemistry, haematology, clotting studies, group \& save and blood cultures), specimen bags, and ABG syringes.

This project runs alongside the NHS Institute for Innovation and Improvement module 'The Productive Ward,' (3) which focuses on improving ward processes and environments to help nurses and therapists spend more time on patient care. The methodology used is derived from the Lean and $5 \mathrm{~S}$ models of quality improvement (4).

\section{Baseline Measurement}

In order to assess the magnitude of the problem, we carried out 
time trials with junior doctors, nursing staff, and physician assistants. We measured how long it took each individual to collect the equipment required to insert a venous cannula on a ward that was most familiar to them ('Home ward') and compared this to the time required to gather the same equipment on an unfamiliar ward ('Away ward'). We compared datasets in order to quantify the effect of familiarity. The mean time on the Home ward was $59 \mathrm{~s}$ (range 20 $\mathrm{s}$ to $2 \min 18 \mathrm{~s}, \mathrm{n}=23$ ) in comparison to the Away ward with a mean of $2 \min 41 \mathrm{~s}$ (range $52 \mathrm{~s}$ to $6 \min 58 \mathrm{~s}, \mathrm{n}=23$ ).

We performed a process map of a junior doctor collecting the equipment to insert a venous cannula on an unfamiliar surgical ward. It took the individual 16 steps ( 1 min $49 \mathrm{~s}$ ) to find the necessary equipment.

We categorised the proximity of pieces of equipment on each ward before our intervention. We took the location of cannulas to be a zero point and then classified each of the items as in the 'same stack', 'adjacent stack', 'non-adjacent stack', or 'different room'. We found there to be no obvious system to the pre-intervention layout; often equipment was located on the opposite sides of the room or even in adjacent rooms. We noted that on two of the four wards we looked at, there were no labelled ABG syringes.

Finally we gathered pre- and post-intervention data from 32 Foundation Year 1 (FY1) doctors via a paper based questionnaire. Candidates used a 10 point scale $(1=$ strongly disagree, $10=$ strongly agree) to rate their opinion of statements relating to time wasted searching for equipment, whether they would support a rearrangement, and whether they felt searching for equipment was ever detrimental to patient care/safety. Candidates also indicated the number of cannulation and venepuncture procedures they performed each day.

See supplementary file: ds2044.pptx - "Initial time trial data (Home vs. Away ward)"

\section{Design}

In order to address the problem of non-uniformity within hospital store rooms, we used a twofold approach. First, we grouped all equipment within as close proximity as possible. We took the cannulas to be a 'zero' point and then ensured that all other equipment was directly adjacent to one another in a logical sequence. The exact location of the grouped items was not uniform across all wards; however, all items required to perform venepuncture, cannulation, and ABG sampling were adjacent to one another. This approach had the added benefit of allowing the model to be changed and adapted to suit different ward store rooms with entirely different layouts.

Of the three procedures we looked at, venous cannulation required the greatest number of individual pieces of equipment and is a common task for the on-call physician. We therefore chose to focus our key quantitative data collection on this particular procedure. Selecting this more time-consuming procedure is likely to show a greater margin of improvement than for the procedures which involve fewer equipment gathering steps.
Our second intervention was to make this location readily identifiable. We did this by using bright red labels ('red dots') on the front of each drawer and/or shelving unit.

\section{Strategy}

PDSA (Plan, Do, Study, Act) cycle 1: A new layout was trialled for 2 weeks on one single surgical ward. Equipment was rearranged to slightly closer proximity (within adjacent cupboards) and all equipment was labelled with writing and a red label to identify the location. This was followed by a feedback from doctors, nurses, and physicians assistants based on that ward. The feedback was largely positive; however, it was felt that the rearrangement should be more extensive. The initial rearrangement caused some confusion as the layout was changed overnight and persons did not immediately understand the purpose of the red dots.

PDSA cycle 2: At the suggestion of the ward sister, all equipment was relocated to one single shelving unit in a set of ascending drawers. This involved minimal displacement of other equipment; only minor rearranging was necessary. Nursing staff, doctors, and physician assistants were educated about the aim of the project and the association of "Taking blood, look for the red dot". Nursing staff then became advocates and promoted the idea.

PDSA cycle 3: After successfully trialling the model on one surgical ward the model was rolled out across the remaining general surgical wards over the next 2 weeks. The system was put in place on the four primary general surgical wards in the hospital.

\section{Post-Measurement}

After rearranging the ward store rooms, we repeated all measurements. The mean time taken to gather a cannula on an unfamiliar ward was reduced from $2 \mathrm{~min} 41 \mathrm{~s}$ to $26 \mathrm{~s}$ (range $8 \mathrm{~s}$ to 1 $\min 20 \mathrm{~s}, \mathrm{n}=51$ ) or $135 \mathrm{~s}$ per cannula. The number of cannulas inserted per day ranged from 1 to 5 with a mean of $2(n=32)$. There were $39 \mathrm{FY} 1$ doctors at St George's Hospital (SGH) equating to 78 cannulas per day inserted by FY1s alone. If this project was rolled out to include all hospital wards the resultant time saved per day would be 175 mins per day of FY1 time alone. We did not collect data on exactly which wards FY1s were inserting cannulas, but focused our intervention on the busy surgical wards.

The proximity of equipment was again re-categorised postintervention. After rearranging the equipment, all items in question, across all four wards, lay directly adjacent to one another. Every FY1 in the survey strongly agreed (score of 10) that 'Faster/easier identification of equipment improves patient safety'. The process mapping of the individual to gather the equipment to insert a venous cannula on a set ward showed a reduction from 16 operations down to five operations. Initially the individual's route involved walking to non-adjacent stacks and searching through drawers looking for equipment. Post-intervention these additional steps were eliminated and on entering the room the individual identified the relevant set of drawers immediately (using the red dot) before sequentially opening the four labelled drawers and finding all required equipment therein. 


\section{Lessons and Limitations}

The most significant barrier to change in this project was the attitudes of staff towards rearranging what was a familiar environment. There was a clear conflict of interest between the staff who worked on one particular ward, who were familiar with the preintervention layout (albeit non-structured), and the on-call staff who were charged with working on a variety of different wards during one shift. We overcame this challenge by engaging key stakeholders at an early stage in the project and allowing ward staff to alter their own environment. It was important to seek endorsement by heads of departments. Senior nurses and the procurement team on each of the wards were consulted beforehand and were key decision makers as to how and where the equipment would be rearranged. This step ensured that disruption to other nursing equipment was minimised and helped to increase awareness of the project dramatically. We found in particular that taking staff away from their Home ward and asking them to search for equipment on unfamiliar wards was an especially powerful tool for highlighting the need for change. We found this was fed back to other staff members almost straight away. During the 2 week trial one staff nurse actually reported "We found less doctors asking us where to find equipment," saving time for both parties and avoiding other team members from being distracted by menial tasks.

We learnt about the nature of data types and collection methods. One difficulty we had was that our data were time-consuming and difficult to obtain. The data collection involved actively taking individuals away from their daily tasks and visiting different wards. We had to be organised with our data collection and to explain the potential value of the projects to earn this time.

Ensuring sustainability once we had rotated on was always going to be challenging. The endorsement by higher level stakeholders, including heads of nursing and procurement, meant that those 'on the shop floor' such as ward stockists, matrons, and nurses valued the project from its inception. Involvement of the nursing staff on the ward in both the implementation and data gathering process proved vital. Junior doctors' frustrations at the previous system ensured their full support of a timely change. For the incoming doctors they will be informed of the system in place during their induction week.

The simplicity of the 'red dot' system greatly minimised disruption and generated little or no extra work for stockists. We contacted the wards 5 months after leaving the hospital, and around 12 months after the initial interventions began. Nursing staff report that the system is still in place and functioning well.

\section{Conclusion}

The original goal of the project was to reduce time wasted by medical staff searching for simple venepuncture, cannulation, and $A B G$ equipment. This had a higher aim of providing more time to spend with patients and in the acute setting, to prevent harm coming to patients as a result of staff wasting time searching for basic equipment.
Our initial findings provided good evidence that the problem was experienced by all staff across all wards. A lack of familiarity with storeroom layouts proved a fundamental barrier to the identification of equipment, more so than the actual location of equipment in the store room. It was here that the red dot system was especially useful.

The reduction in time taken to collect cannulation equipment showed a significant improvement following our project. There is no reason why the system should not be extended across the Trust.

\section{References}

1. Chan P, Krumholz H, Nichol G, et al. Delayed time to defibrillation after in-hospital cardiac arrest. N Engl J Med 2008;358:9-17.

2. Royal College of Anaesthetists, Royal College of Physicians of London, Intensive Care Society, Resuscitation Council (UK). Cardiopulmonary resuscitation: standards for clinical practice and training. Resuscitation Council (UK). Published October 2004, updated June 2008.

3. NHS Institute for Innovation and Improvement. The Productive Ward: Releasing time to care. http://www.institute.nhs.uk. (accessed 21 April 2013)

4. NHS Institute for Innovation and Improvement. Quality and service improvement tools. Lean. http://www.institute.nhs.uk . (accessed 21 April 2013)

\section{Declaration of interests}

Nothing to declare

\section{Acknowledgements}

Dr Jessica Wynter-Bee (FY2, Frimley Park), Alison Murphy (Head of Procurement, SGH), Claire Painter (Head of Nursing, SGH), and Jenny Muir (Head of Nursing Corporate, SGH) 